\section{Economic Analysis of the M/M/1/N Queuing System Cost Model in a Vague Environment}

\section{Hamed Fazlollahtabar ${ }^{1}$ and Hadi Gholizadeh ${ }^{2}$}

${ }^{1}$ Department of Industrial Engineering, School of Engineering, Damghan University, Damghan, Iran

${ }^{2}$ Department of Industrial Engineering, Mazandaran University of Science and Technology, Babol, Iran

\begin{abstract}
In the real world, values are not always accurate, since vague and uncertain data exist in many applications. In this paper, a single-server finite capacity Markovian queuing system being called $\mathrm{M} / \mathrm{M} / 1 / \mathrm{N}$ with encouraged arrivals is considered. The different arrival rates, service rates and processing times of labor/duties are usually supposed to be uncertain. Therefore, we describe the theory of queuing in a vague environment in which encourage arrivals rates and service rates are considered to be vague numbers. Then, economic analysis of the model is presented by developing a cost model proceeding to exert uncertainty of the primary information when some of the parameters of the models are vague.
\end{abstract}

Keywords: Encouraged arrivals rate, Queuing system, Vague environment, Vague numbers

\section{Introduction}

The study of waiting lines, called queuing theory, is one of the oldest and most widely used quantitative analysis techniques. Queuing theory is basically a mathematical approach, used for the analysis of expecting lines. One of the applications of queuing theory is to analyze the congeries and delay of waiting in line.

Queuing theory has a long background and has been handle to decide applied problems in manufacturing, communication, inventory control, etc. Queuing system is composed of three parts (1) the arrival or inputs to the system (sometimes referred to as the calling population), (2) the queue (or the waiting line itself), and (3) the service facility. In the queuing theory, it is usually supposed that the time between the two successively arrivals and the servicing time follow a particular probability distribution. However, in the real world, this kind of information is gained using qualitative data being expressed by words equal rapid, middle, and slow rather than the probability distribution. It can be said that fuzzy queuing models are more realistic and practical than the classical ones. Queuing theory has its beginning in the research work of a Danish engineer [1]. applied this theory extensively to study the manner of phone networks. Since in the actual world there is vague information about different applications, such as in sensor databases, we can formalize the measurements from different sensors to a vague set in the real world. The various arrival rates, service rates and processing times of jobs/tasks usually assumed to be uncertainty. Thus, fuzzy set theory has been suggested to batch such ambiguousness by distribute the concept of membership in a set. Fuzzy queuing models have been explained by several scholar, namely Li and Lee [2], Buckley [3], Negi and Lee [4], Kao et al. [5], Chen [6, 7] who dragged fuzzy queues using 
using Zadeh's deployment origin [8]. Kao et al. [5] created the membership functions of the system characteristic for fuzzy queues using parametric linear programming. Basically, in a fuzzy set each base is related with a point value opted from the unit interval $[0,1]$, A vague set, as well as an Intuitionistic fuzzy set, is a further extension of a fuzzy set. Instead of using point-relying membership as in fuzzy sets, interval-based membership is used in a vague set [9]. Defined vague sets are used by Gonzalez-Lopez et al. [10] where they described the optimization of queuing theory based on vague environment.

Oz et al. [11] considered the $\mathrm{M} n / \mathrm{G} n / 1$ queue with vacations and exhaustive service in which the server takes (repeated) vacations whenever it becomes idle, the service time distribution is queue length dependent, and the arrival rate varies both with the queue length and with the status of the server, being busy or on vacation. Dutkova et al. [12] displayed the usage of simulation method as a tool for optimizing costs of particular post office. Simulation methods make it possible to eliminate the most of the obstacles that arise from the analytical solution of queuing systems such as queuing system of post office.

Hanczewski et al. [13] presented a multi-service model of a queueing system with elastic and adaptive traffic. The presented model permits to determine the characteristics of a multi-service queueing system, such as blocking probability or the average queueing length.

Legros [14] analyzed the transient behavior of the M/M/1+D queue. Considering an Erlang distribution for customers' waiting time, he approximated the real system by a Markov chain. Then, obtained the Laplace transform of the transient probabilities in the approximated model and the Laplace transform of the main performance measures for the real system.

Here, the attracted customers are entitling as encourage arrivals by the obscure rate. The concept of customer mobilization was famous by [15]. They called it inverse balking. The phenomenon of encouraged arrivals can also be understood as opposite to disheartened arrivals bring up by [16]. Explain the $\mathrm{M} / \mathrm{M} / 1 / \mathrm{N}$ queuing system with encouraged arrivals [17].

Literature review shows that no researchers have discussed the problem of a single server with limited capacity in the $\mathrm{M} / \mathrm{M} / 1 \mathrm{~N}$ Markovian system with vague encouraged inputs. In real, the input data, arrival rate and service rate are uncertain. Uncertainty is resolved by using vague set theory. So, the classical queuing model will be more effective if it is proposed using vague models.

As a result, the major contribution of the work is to develop an $\mathrm{M} / \mathrm{M} / 1 / \mathrm{N}$ queue model using vague numbers and as a minor contribution, the corresponding economic analysis through a novel cost model is proposed.

The remainder of the paper is organized as follows: in Section 2, the $\mathrm{M} / \mathrm{M} / 1 / \mathrm{N}$ queue model is introduced. In Section 3, various concepts of the vague set theory are discussed. The vague queue model $\mathrm{M} / \mathrm{M} / 1 / \mathrm{N}$ is introduced in Section 4. In Section 5, the analysis of the model is expressed. In Section 6, a numerical example is illustrated to show the effectiveness of the proposed model. We conclude in Section 7.

\section{M/M/1/N Queue Model}

In this model, the customers arrive based on the Poisson distribution with rate $(1+\eta)$ and receive service which is exponentially distributed with a mean service time of $\mu$ from a single server and the capacity of the system is finite say, $N$ [17].

We first outline the assumptions of the problem:

- Customers arrive based on the Poisson distribution with parameter $(1+\eta)$, where ' $~ \eta$ ' displays the percent change in the number of customers computed from bygone or perceives data. That is $\lambda$, vague.

- We show the service rate with $\mu$ that is vague.

- The capacity of the system is finite say, $N$.

Due to the definition and assumption the following queue model computation are given.

The possibility of system being empty is computed as follows [17]:

$$
\begin{aligned}
P_{0} & =\operatorname{Pr}\{\text { system is empty }\} \\
& =\left\{1+\sum_{n=1}^{N}\left[\frac{\lambda(1+\eta)}{\mu}\right]^{n}\right\}^{-1}=\frac{1-\left(\frac{\lambda(1+\eta)}{\mu}\right)}{1-\left(\frac{\lambda(1+\eta)}{\mu}\right)^{N+1}} .
\end{aligned}
$$

And the probability that system is full is given by [17]:

$$
\begin{aligned}
P_{N} & =\operatorname{Pr}\{\text { system is full }\} \\
& =\left[\frac{\lambda(1+\eta)}{\mu}\right]^{N} *\left(\frac{1-\left(\frac{\lambda(1+\eta)}{\mu}\right)}{1-\left(\frac{\lambda(1+\eta)}{\mu}\right)^{N+1}}\right) .
\end{aligned}
$$

Expected number of customers in the system [12]:

$$
L=\sum_{n=1}^{N} n\left[\frac{\lambda(1+\eta)}{\mu}\right]^{n} *\left(\frac{1-\left(\frac{\lambda(1+\eta)}{\mu}\right)}{1-\left(\frac{\lambda(1+\eta)}{\mu}\right)^{N+1}}\right) .
$$


Expected number of the customers waiting on the queue [17]:

$$
L_{q}=\sum_{n=1}^{N}(n-1)\left[\frac{\lambda(1+\eta)}{\mu}\right]^{n} *\left(\frac{1-\left(\frac{\lambda(1+\eta)}{\mu}\right)}{1-\left(\frac{\lambda(1+\eta)}{\mu}\right)^{N+1}}\right) .
$$

Expected waiting time of customers in the system [17]:

$$
W=\frac{L}{\lambda}, W_{q}=\frac{L_{q}}{\lambda} .
$$

In the special case if $\eta=0$ of that time [17]:

$$
\begin{aligned}
& P_{n}=\operatorname{Pr}\{\text { system is full }\}=\left(\frac{\lambda}{\mu}\right)^{N} P_{n}=\rho^{N} P_{0}, \\
& P_{0}=\operatorname{Pr}\{\text { system is empty }\}=\frac{1-\left(\frac{\lambda}{\mu}\right)}{1-\left(\frac{\lambda}{\mu}\right)^{N+1}}=\frac{1-\rho}{1-\rho^{N+1}},
\end{aligned}
$$

where $\rho=\frac{\lambda}{\mu}<1$, is the traffic intensity.

\section{Vague Environment}

To conceptualize the vagueness, some preliminary definitions one presented below:

Definition 1 (Fuzzy set) [10]. A fuzzy set $\tilde{A}=\{<u$, $\left.\mu_{\tilde{A}}(u)>\mid u \in U\right\}$ in a universe of discourse $U$ is characterized by a membership function, $\mu_{\tilde{A}}$, as follows:

$$
\mu_{\tilde{A}}: U \rightarrow[0,1] .
$$

Definition 2 [10]. A vague set $\tilde{A}$ in a universe of discourse $U$ is characterized It is expressed by two membership functions, which is a true membership, $T_{\tilde{A}}$, function and a false membership function, $F_{\tilde{A}}$, as follows: $T_{\tilde{A}}: U \rightarrow[0,1], F_{\tilde{A}}: U \rightarrow$ $[0,1]$, and $T_{\tilde{A}}(u)+F_{\tilde{A}}(u) \leq 1$, where $T_{\tilde{A}}(u)$ is a lower bound on the grade of membership of $u$ derived from the evidence for $u, F_{\tilde{A}}(u)$ is a lower bound on the grade of membership of the negation of $u$ derived from the evidence against $u$. Suppose $U=\left\{u_{1}, u_{2}, \ldots, u_{n}\right\}$. A vague set $\tilde{A}$ of the universe of discourse $U$ can be represented by $\tilde{A}=\frac{\sum_{i=1}^{N}\left[T_{\tilde{A}}\left(u_{i}\right), 1-F_{\tilde{A}}\left(u_{i}\right)\right]}{u_{i}}$, where $0 \leq T\left(u_{i}\right) \leq 1-F\left(u_{i}\right) \leq 1$ and $1 \leq i \leq n$. In this case, the degree of membership in $u_{i}$ will be limited to a period of time, $\left[T_{\tilde{A}}\left(u_{i}\right), 1-F_{\tilde{A}}\left(u_{i}\right)\right]$ Of $[0,1]$.

Therefore, generalized vague collections have fuzzy sets of membership degrees $\mu_{\tilde{A}}(u)$ of $u$ in Definition 1 may be inexact in a vague set.
Definition $3[10]$. The complement of a vague set $\tilde{A}$ is denoted by $\tilde{A}^{\prime}$ and is defined by

$$
T_{\tilde{A}^{\prime}}(u)=F_{\tilde{A}}(u), 1-F_{\tilde{A}^{\prime}}(u)=1-F_{\tilde{A}}(u) .
$$

Definition 4 [10]. Let $\tilde{A}_{T_{\tilde{A}} F_{\tilde{A}}}$ be a vague set of $U$. Then, we define $\alpha_{T}$-cuts and $\alpha_{F}$-cuts of $\tilde{A}$ as the crisp sets of $U$ given by

$$
\begin{aligned}
\tilde{A}_{\alpha T} & =\left\{u: T_{\tilde{A}}(u) \geq \alpha_{T}\right\}, & & \alpha_{T} \in[0,1], \\
\tilde{A}_{\alpha F} & =\left\{u: F_{\tilde{A}}(u) \geq \alpha_{F}\right\}, & & \alpha_{F} \in[0,1] .
\end{aligned}
$$

Definition $5\left[10 \mid\right.$. A vague set $\tilde{A}_{T_{\tilde{A}} F_{\tilde{A}}}$ of $\mathcal{R}$ with continuous membership functions $T_{\tilde{A}}$ and $F_{\tilde{A}}$ is called a vague number if and only if $\tilde{A}_{\alpha T}$ and $\tilde{A}_{\alpha F}$, for all $\alpha_{T}, \alpha_{F} \in(0,1]$, are bounded closed intervals; i.e.,

$$
\tilde{A}_{\alpha T}=\left[\tilde{A}_{\alpha T}^{L}, \tilde{A}_{\alpha T}^{U}\right], \quad \tilde{A}_{\alpha F}=\left[\tilde{A}_{\alpha F}^{L}, \tilde{A}_{\alpha F}^{U}\right] .
$$

We denote the class of all vague numbers by $\mathrm{A}(\mathcal{R})$.

Definition $6[10]$. The vague number $\tilde{A}_{T_{\tilde{A}} F_{\tilde{A}}}$ is called a trapezoidal vague number, if

$$
\begin{gathered}
T_{\tilde{A}}(u)= \begin{cases}\frac{u-a_{1}}{w\left(a_{2}-a_{1}\right)}, & a_{1} \leq u \leq a_{2}, \\
1, & a_{2} \leq u \leq a_{3}, \\
\frac{a_{4}-u}{w\left(a_{4}-a_{3}\right)}, & a_{3} \leq u \leq a_{4} . \\
0, & \text { otherwise, }\end{cases} \\
1-F_{\tilde{A}}(u)= \begin{cases}\frac{u-a_{1}}{\left(a_{2}-a_{1}\right)}, & a_{1} \leq u \leq a_{2}, \\
\frac{a_{4}-u}{\left(a_{4}-a_{3}\right)}, & a_{3} \leq u \leq a_{3}, \\
0, & \text { otherwise },\end{cases}
\end{gathered}
$$

where $w \in[1, \infty)$. We denote such a vague number by $\tilde{A}=$ $\left(a_{1}, a_{2}, a_{3}, a_{4}, w\right)_{T}$.

\subsection{Vague Queues}

This paper develops vague queuing $\mathrm{M} / \mathrm{M} / 1 / \mathrm{N}$ model in which the source encouraged arrivals rate and service rate and the percentage of change in the number of customers are uncertain parameters.

Since the arrival rate $(\tilde{\lambda})$, service rate $(\tilde{\mu})$ and the percentage of change in number of customers $(\tilde{\eta})$ are not known precisely, therefore, we use trapezoidal vague numbers to represent them. Let $\tilde{\lambda}=\left(\lambda_{1}, \lambda_{2}, \lambda_{3}, \lambda_{4}\right), \tilde{\mu}=\left(\mu_{1}, \mu_{2}, \mu_{3}, \mu_{4}\right)$, 
$\tilde{\eta}=\left(\eta_{1}, \eta_{2}, \eta_{3}, \eta_{4}\right)$, where $\lambda_{1} \leq \lambda_{2} \leq \lambda_{3} \leq \lambda_{4}, \mu_{1} \leq \mu_{2} \leq$ $\mu_{3} \leq \mu_{4}, \eta_{1} \leq \eta_{2} \leq \eta_{3} \leq \eta_{4}$ based on subjective judgment. The membership of $T_{\tilde{\lambda}}(\lambda), 1-F_{\tilde{\lambda}}(\lambda)$ and $T_{\tilde{\mu}}(\mu), 1-F_{\tilde{\mu}}(\mu)$ and $T_{\tilde{\eta}}(\eta), 1-F_{\tilde{\eta}}(\eta)$ are defined as follows:

$$
T_{\tilde{\lambda}}(\lambda)= \begin{cases}\frac{\lambda-\lambda_{1}}{w\left(\lambda_{2}-\lambda_{1}\right)}, & \lambda_{1} \leq \lambda \leq \lambda_{2}, \\ 1, & \lambda_{2} \leq \lambda \leq \lambda_{3}, \\ \frac{\lambda_{4}-\lambda}{w\left(\lambda_{4}-\lambda_{3}\right)}, & \lambda_{3} \leq \lambda \leq \lambda_{4}, \\ 0, & \text { otherwise, }\end{cases}
$$$$
1-F_{\tilde{\lambda}}(\lambda)= \begin{cases}\frac{\lambda-\lambda_{1}}{\left(\lambda_{2}-\lambda_{1}\right)}, & \lambda_{1} \leq \lambda \leq \lambda_{2}, \\ 1, & \lambda_{2} \leq \lambda \leq \lambda_{3}, \\ \frac{\lambda_{4}-\lambda}{\left(\lambda_{4}-\lambda_{3}\right)}, & \lambda_{3} \leq \lambda \leq \lambda_{4}, \\ 0, & \text { otherwise, }\end{cases}
$$$$
T_{\tilde{\mu}}(\mu)= \begin{cases}\frac{\mu-\mu_{1}}{w\left(\mu_{2}-\mu_{1}\right)}, & \mu_{1} \leq \mu \leq \mu_{2}, \\ 1, & \mu_{2} \leq \mu \leq \mu_{3}, \\ \frac{\mu_{4}-\mu}{w\left(\mu_{4}-\mu_{3}\right)}, & \mu_{3} \leq \mu \leq \mu_{4}, \\ 0, & \text { otherwise, }\end{cases}
$$$$
1-F_{\tilde{\mu}}(\mu)= \begin{cases}\frac{\mu-\mu_{1}}{\left(\mu_{2}-\mu_{1}\right)}, & \mu_{1} \leq \mu \leq \mu_{2}, \\ 1, & \mu_{2} \leq \mu \leq \mu_{3}, \\ \frac{\mu_{4}-\mu}{\left(\mu_{4}-\mu_{3}\right)}, & \mu_{3} \leq \mu \leq \mu_{4}, \\ 0, & \text { otherwise, }\end{cases}
$$$$
T_{\tilde{\eta}}(\eta)= \begin{cases}\frac{\eta-\eta_{1}}{w\left(\eta_{2}-\eta_{1}\right)}, & \eta_{1} \leq \eta \leq \eta_{2}, \\ 1, & \eta_{2} \leq \eta \leq \eta_{3}, \\ \frac{\eta_{4}-\eta}{w\left(\eta_{4}-\eta_{3}\right)}, & \eta_{3} \leq \eta \leq \eta_{4}, \\ 0, & \text { otherwise }\end{cases}
$$$$
1-F_{\tilde{\eta}}(\eta)= \begin{cases}\frac{\eta-\eta_{1}}{\left(\eta_{2}-\eta_{1}\right)}, & \eta_{1} \leq \eta \leq \eta_{2}, \\ 1, & \eta_{2} \leq \eta \leq \eta_{3}, \\ \frac{\eta_{4}-\eta}{\left(\eta_{4}-\eta_{3}\right)}, & \eta_{3} \leq \eta \leq \eta_{4}, \\ 0, & \text { otherwise. }\end{cases}
$$

Using the concept of $\alpha$-cut method, we have

$$
\frac{\tilde{\lambda}-\lambda_{1}}{w\left(\lambda_{2}-\lambda_{1}\right)}=\alpha_{T} \Longrightarrow \tilde{\lambda}=w \alpha_{T}\left(\lambda_{2}-\lambda_{1}\right)+\lambda_{1}
$$

$$
\begin{aligned}
& \frac{\lambda_{4}-\tilde{\lambda}}{w\left(\lambda_{4}-\lambda_{3}\right)}=\alpha_{T} \Longrightarrow \tilde{\lambda}=\lambda_{4}-w \alpha_{T}\left(\lambda_{4}-\lambda_{3}\right), \\
& \quad \tilde{\lambda}_{\alpha_{T}}=\left[w \alpha_{T}\left(\lambda_{2}-\lambda_{1}\right)+\lambda_{1}, \lambda_{4}-w \alpha_{T}\left(\lambda_{4}-\lambda_{3}\right)\right],
\end{aligned}
$$

$$
\begin{aligned}
& \frac{\tilde{\lambda}-\lambda_{1}}{\left(\lambda_{2}-\lambda_{1}\right)}=\alpha_{F} \Longrightarrow \tilde{\lambda}=\alpha_{F}\left(\lambda_{2}-\lambda_{1}\right)+\lambda_{1}, \\
& \frac{\lambda_{4}-\tilde{\lambda}}{\left(\lambda_{4}-\lambda_{3}\right)}=\alpha_{F} \Longrightarrow \tilde{\lambda}=\lambda_{4}-\alpha_{F}\left(\lambda_{4}-\lambda_{3}\right), \\
& \tilde{\lambda}_{\alpha_{F}}=\left[\alpha_{F}\left(\lambda_{2}-\lambda_{1}\right)+\lambda_{1}, \lambda_{4}-\alpha_{F}\left(\lambda_{4}-\lambda_{3}\right)\right],(21) \\
& \frac{\tilde{\mu}-\mu_{1}}{w\left(\mu_{2}-\mu_{1}\right)}=\alpha_{T} \Longrightarrow \tilde{\mu}=w \alpha_{T}\left(\mu_{2}-\mu_{1}\right)+\mu_{1}, \\
& \frac{\mu_{4}-\tilde{\mu}}{w\left(\mu_{4}-\mu_{3}\right)}=\alpha_{T} \Longrightarrow \tilde{\mu}=\mu_{4}-w \alpha_{T}\left(\mu_{4}-\mu_{3}\right), \\
& \tilde{\mu}_{\alpha_{T}}=\left[w \alpha_{T}\left(\mu_{2}-\mu_{1}\right)+\mu_{1}, \mu_{4}-w \alpha_{T}\left(\mu_{4}-\mu_{3}\right)\right],
\end{aligned}
$$

$$
\begin{aligned}
\frac{\tilde{\mu}-\mu_{1}}{\left(\mu_{2}-\mu_{1}\right)} & =\alpha_{F} \Longrightarrow \tilde{\mu}=\alpha_{F}\left(\mu_{2}-\mu_{1}\right)+\mu_{1}, \\
\frac{\mu_{4}-\tilde{\mu}}{\left(\mu_{4}-\mu_{1}\right)} & =\alpha_{F} \Longrightarrow \tilde{\mu}=\mu_{4}-\alpha_{F}\left(\mu_{4}-\mu_{3}\right), \\
\tilde{\mu}_{\alpha_{F}} & =\left[\alpha_{F}\left(\mu_{2}-\mu_{1}\right)+\mu_{1}, \mu_{4}-\alpha_{F}\left(\mu_{4}-\mu_{3}\right)\right],
\end{aligned}
$$

$$
\begin{aligned}
& \frac{\tilde{\eta}-\eta_{1}}{w\left(\eta_{2}-\eta_{1}\right)}=\alpha_{T} \Longrightarrow \tilde{\eta}=w \alpha_{T}\left(\eta_{2}-\eta_{1}\right)+\eta_{1}, \\
& \frac{\eta_{4}-\tilde{\eta}}{w\left(\eta_{4}-\eta_{3}\right)}=\alpha_{T} \Longrightarrow \tilde{\eta}=\eta_{4}-w \alpha_{T}\left(\eta_{4}-\eta_{3}\right), \\
& \tilde{\eta}_{\alpha_{T}}=\left[w \alpha_{T}\left(\eta_{2}-\eta_{1}\right)+\eta_{1}, \eta_{4}-w \alpha_{T}\left(\eta_{4}-\eta_{3}\right)\right],
\end{aligned}
$$

$$
\begin{aligned}
\frac{\tilde{\eta}-\eta_{1}}{\left(\eta_{2}-\eta_{1}\right)} & =\alpha_{F} \Longrightarrow \tilde{\eta}=\alpha_{F}\left(\eta_{2}-\eta_{1}\right)+\eta_{1}, \\
\frac{\eta_{4}-\tilde{\eta}}{\left(\eta_{4}-\eta_{3}\right)} & =\alpha_{F} \Longrightarrow \tilde{\eta}=\eta_{4}-\alpha_{F}\left(\eta_{4}-\eta_{3}\right), \\
\tilde{\eta}_{\alpha_{F}} & =\left[\alpha_{F}\left(\eta_{2}-\eta_{1}\right)+\eta_{1}, \eta_{4}-\alpha_{F}\left(\eta_{4}-\eta_{3}\right)\right] .
\end{aligned}
$$

Then, $\tilde{\lambda}_{\alpha_{T}}, \tilde{\lambda}_{\alpha_{F}}$ and $\tilde{\mu}_{\alpha_{T}}, \tilde{\mu}_{\alpha_{F}}$ and $\tilde{\eta}_{\alpha_{T}}, \tilde{\eta}_{\alpha_{F}}$ are vague numbers.

$$
\begin{aligned}
& \tilde{\lambda}_{\alpha_{T}}=\left[\tilde{\lambda}_{\alpha_{T}}^{L}, \tilde{\lambda}_{\alpha_{T}}^{U}\right], \tilde{\lambda}_{\alpha_{F}}=\left[\tilde{\lambda}_{\alpha_{F}}^{L}, \tilde{\lambda}_{\alpha_{F}}^{U}\right], \\
& \tilde{\mu}_{\alpha_{T}}=\left[\tilde{\mu}_{\alpha_{T}}^{L}, \tilde{\mu}_{\alpha_{T}}^{U}\right], \tilde{\mu}_{\alpha_{F}}=\left[\tilde{\mu}_{\alpha_{F}}^{L}, \tilde{\mu}_{\alpha_{F}}^{U}\right] \text {, } \\
& \tilde{\eta}_{\alpha_{T}}=\left[\tilde{\eta}_{\alpha_{T}}^{L}, \tilde{\eta}_{\alpha_{T}}^{U}\right], \quad \tilde{\eta}_{\alpha_{F}}=\left[\tilde{\eta}_{\alpha_{F}}^{L}, \tilde{\eta}_{\alpha_{F}}^{U}\right] .
\end{aligned}
$$

\section{Vague $M / M / 1 / N$ Model}

Given what have explained above, we'll analyze M/M/1/N model under vague environment. 
Vague empty system:

$$
\begin{aligned}
& \left(\tilde{\mathrm{P}_{0}}\right)_{\alpha_{T}}^{L}=\frac{1-\left(\frac{\lambda_{\alpha_{T}}^{L}\left(1+\eta_{\alpha_{T}}^{L}\right)}{\mu_{\alpha_{T}}^{L}}\right)}{1-\left(\frac{\lambda_{\alpha_{T}}^{L}\left(1+\eta_{\alpha_{T}}^{L}\right)}{\mu_{\alpha_{T}}^{L}}\right)^{N+1}} \\
& =\frac{1-\left(\frac{\left(w \alpha_{T}\left(\lambda_{2}-\lambda_{1}\right)+\lambda_{1}\right)\left(1+\left(w \alpha_{T}\left(\eta_{2}-\eta_{1}\right)+\eta_{1}\right)\right)}{\left(w \alpha_{T}\left(\mu_{2}-\mu_{1}\right)+\mu_{1}\right)}\right)}{1-\left(\frac{\left(w \alpha_{T}\left(\lambda_{2}-\lambda_{1}\right)+\lambda_{1}\right)\left(1+\left(w \alpha_{T}\left(\eta_{2}-\eta_{1}\right)+\eta_{1}\right)\right)}{\left(w \alpha_{T}\left(\mu_{2}-\mu_{1}\right)+\mu_{1}\right)}\right)^{N+1}}, \\
& \left(\tilde{\mathrm{P}_{0}}\right)_{\alpha_{T}}^{U}=\frac{1-\left(\frac{\lambda_{\alpha_{T}}^{U}\left(1+\eta_{\alpha_{T}}^{U}\right)}{\mu_{\alpha_{T}}^{U}}\right)}{1-\left(\frac{\lambda_{\alpha_{T}}^{U}\left(1+\eta_{\alpha_{T}}^{U}\right)}{\mu_{\alpha_{T}}^{U}}\right)^{N+1}} \\
& =\frac{1-\left(\frac{\left(\lambda_{4}-w \alpha_{T}\left(\lambda_{4}-\lambda_{3}\right)\right)\left(1+\left(\eta_{4}-w \alpha_{T}\left(\eta_{4}-\eta_{3}\right)\right)\right)}{\left(\mu_{4}-w \alpha_{T}\left(\mu_{4}-\mu_{3}\right)\right)}\right)}{1-\left(\frac{\left(\lambda_{4}-w \alpha_{T}\left(\lambda_{4}-\lambda_{3}\right)\right)\left(1+\left(\eta_{4}-w \alpha_{T}\left(\eta_{4}-\eta_{3}\right)\right)\right)}{\left(\mu_{4}-w \alpha_{T}\left(\mu_{4}-\mu_{3}\right)\right)}\right)^{N+1}}, \\
& \left(\tilde{\mathrm{P}_{0}}\right)_{\alpha_{F}}^{L}=\frac{1-\left(\frac{\lambda_{\alpha_{F}}^{L}\left(1+\eta_{\alpha_{F}}^{L}\right)}{\mu_{\alpha_{F}}^{L}}\right)}{1-\left(\frac{\lambda_{\alpha_{F}}^{L}\left(1+\eta_{\alpha_{F}}^{L}\right)}{\mu_{\alpha_{F}}^{L}}\right)^{N+1}} \\
& =\frac{1-\left(\frac{\left(\alpha_{F}\left(\lambda_{2}-\lambda_{1}\right)+\lambda_{1}\right)\left(1+\left(\alpha_{F}\left(\eta_{2}-\eta_{1}\right)+\eta_{1}\right)\right)}{\left(\alpha_{F}\left(\mu_{2}-\mu_{1}\right)+\mu_{1}\right)}\right)}{1-\left(\frac{\left(\alpha_{F}\left(\lambda_{2}-\lambda_{1}\right)+\lambda_{1}\right)\left(1+\left(\alpha_{F}\left(\eta_{2}-\eta_{1}\right)+\eta_{1}\right)\right)}{\left(\alpha_{F}\left(\mu_{2}-\mu_{1}\right)+\mu_{1}\right)}\right)^{N+1}}, \\
& \left(\tilde{\mathrm{P}_{0}}\right)_{\alpha_{F}}^{U}=\frac{1-\left(\frac{\lambda_{\alpha_{F}}^{U}\left(1+\eta_{\alpha_{F}}^{U}\right)}{\mu_{\alpha_{F}}^{U}}\right)}{1-\left(\frac{\lambda_{\alpha_{F}}^{U}\left(1+\eta_{\alpha_{F}}^{U}\right)}{\mu_{\alpha_{F}}^{U}}\right)^{N+1}} \\
& =\frac{1-\left(\frac{\left(\lambda_{4}-\alpha_{F}\left(\lambda_{4}-\lambda_{3}\right)\right)\left(1+\left(\eta_{4}-\alpha_{F}\left(\eta_{4}-\eta_{3}\right)\right)\right)}{\left(\mu_{4}-\alpha_{F}\left(\mu_{4}-\mu_{3}\right)\right)}\right)}{1-\left(\frac{\left(\lambda_{4}-\alpha_{F}\left(\lambda_{4}-\lambda_{3}\right)\right)\left(1+\left(\eta_{4}-\alpha_{F}\left(\eta_{4}-\eta_{3}\right)\right)\right)}{\left(\mu_{4}-\alpha_{F}\left(\mu_{4}-\mu_{3}\right)\right)}\right)^{N+1}} .
\end{aligned}
$$

Vague probability that system is full is given by:

$$
\begin{aligned}
\left(\tilde{P}_{N}\right)_{\alpha_{T}}^{L} & {\left[\frac{\lambda_{\alpha_{T}}^{L}\left(1+\eta_{\alpha_{T}}^{L}\right)}{\mu_{\alpha_{T}}^{L}}\right]^{N} *\left(\frac{1-\left(\frac{\lambda_{\alpha_{T}}^{L}\left(1+\eta_{\alpha_{T}}^{L}\right)}{\mu_{\alpha_{T}}^{L}}\right)}{1-\left(\frac{\lambda_{\alpha_{T}}^{L}\left(1+\eta_{\alpha_{T}}^{L}\right)}{\mu_{\alpha_{T}}^{L}}\right)^{N+1}}\right) } \\
= & {\left[\left(\frac{\left(w \alpha_{T}\left(\lambda_{2}-\lambda_{1}\right)+\lambda_{1}\right)\left(1+\left(w \alpha_{T}\left(\eta_{2}-\eta_{1}\right)+\eta_{1}\right)\right)}{\left(w \alpha_{T}\left(\mu_{2}-\mu_{1}\right)+\mu_{1}\right)}\right)\right]^{N} } \\
& *\left(\frac{1-\left(\frac{\left(w \alpha_{T}\left(\lambda_{2} \lambda_{1}\right)+\lambda_{1}\right)\left(1+\left(w \alpha_{T}\left(\eta_{2} \eta_{1}\right)+\eta_{1}\right)\right)}{\left(w \alpha_{T}\left(\mu_{2} \mu_{1}\right)+\mu_{1}\right)}\right)}{1-\left(\frac{\left(w \alpha_{T}\left(\lambda_{2} \lambda_{1}\right)+\lambda_{1}\right)\left(1+\left(w \alpha_{T}\left(\eta_{2}-\eta_{1}\right)+\eta_{1}\right)\right)}{\left(w \alpha_{T}\left(\mu_{2} \mu_{1}\right)+\mu_{1}\right)}\right)^{N+1}}\right),
\end{aligned}
$$

$$
\begin{aligned}
& \left(\tilde{P}_{N}\right)_{\alpha_{T}}^{U} \\
& =\left[\frac{\lambda_{\alpha_{T}}^{U}\left(1+\eta_{\alpha_{T}}^{U}\right)}{\mu_{\alpha_{T}}^{U}}\right]^{N} *\left(\frac{1-\left(\frac{\lambda_{\alpha_{T}}^{U}\left(1+\eta_{\alpha_{T}}^{U}\right)}{\mu_{\alpha_{T}}^{U}}\right)}{1-\left(\frac{\lambda_{\alpha_{T}}^{U}\left(1+\eta_{\alpha_{T}}^{U}\right)}{\mu_{\alpha_{T}}^{U}}\right)^{N+1}}\right) \\
& =\left[\left(\frac{\left(\lambda_{4}-w \alpha_{T}\left(\lambda_{4}-\lambda_{3}\right)\right)\left(1+\left(\eta_{4}-w \alpha_{T}\left(\eta_{4}-\eta_{3}\right)\right)\right)}{\left(\mu_{4}-w \alpha_{T}\left(\mu_{4}-\mu_{3}\right)\right)}\right)\right]^{N} \\
& \quad *\left(\frac{1-\left(\frac{\left(\lambda_{4}-w \alpha_{T}\left(\lambda_{4}-\lambda_{3}\right)\right)\left(1+\left(\eta_{4}-w \alpha_{T}\left(\eta_{4}-\eta_{3}\right)\right)\right)}{\left(\mu_{4}-w \alpha_{T}\left(\mu_{4}-\mu_{3}\right)\right)}\right)}{1-\left(\frac{\left(\lambda_{4}-w \alpha_{T}\left(\lambda_{4}-\lambda_{3}\right)\right)\left(1+\left(\eta_{4}-w \alpha_{T}\left(\eta_{4}-\eta_{3}\right)\right)\right)}{\left(\mu_{4}-w \alpha_{T}\left(\mu_{4}-\mu_{3}\right)\right)}\right)^{N+1}}\right),
\end{aligned}
$$$$
\left(\tilde{P}_{N}\right)_{\alpha_{F}}^{L}
$$$$
=\left[\frac{\lambda_{\alpha_{F}}^{L}\left(1+\eta_{\alpha_{F}}^{L}\right)}{\mu_{\alpha_{F}}^{L}}\right]^{N} *\left(\frac{1-\left(\frac{\lambda_{\alpha_{F}}^{L}\left(1+\eta_{\alpha_{F}}^{L}\right)}{\mu_{\alpha_{F}}^{L}}\right)}{1-\left(\frac{\lambda_{\alpha_{F}}^{L}\left(1+\eta_{\alpha_{F}}^{L}\right)}{\mu_{\alpha_{F}}^{L}}\right)^{N+1}}\right)
$$$$
=\left[\left(\frac{\left(\alpha_{F}\left(\lambda_{2}-\lambda_{1}\right)+\lambda_{1}\right)\left(1+\left(\alpha_{F}\left(\eta_{2}-\eta_{1}\right)+\eta_{1}\right)\right)}{\left(\alpha_{F}\left(\mu_{2}-\mu_{1}\right)+\mu_{1}\right)}\right)\right]^{N}
$$$$
\text { * }\left(\frac{1-\left(\frac{\left(\alpha_{F}\left(\lambda_{2}-\lambda_{1}\right)+\lambda_{1}\right)\left(1+\left(\alpha_{F}\left(\eta_{2}-\eta_{1}\right)+\eta_{1}\right)\right)}{\left(\alpha_{F}\left(\mu_{2}-\mu_{1}\right)+\mu_{1}\right)}\right)}{1-\left(\frac{\left(\alpha_{F}\left(\lambda_{2}-\lambda_{1}\right)+\lambda_{1}\right)\left(1+\left(\alpha_{F}\left(\eta_{2}-\eta_{1}\right)+\eta_{1}\right)\right)}{\left(\alpha_{F}\left(\mu_{2}-\mu_{1}\right)+\mu_{1}\right)}\right)^{N+1}}\right),
$$

$$
\begin{aligned}
& \left(\tilde{P}_{N}\right)_{\alpha_{F}}^{U} \\
& =\left[\frac{\lambda_{\alpha_{F}}^{U}\left(1+\eta_{\alpha_{F}}^{U}\right)}{\mu_{\alpha_{F}}^{U}}\right]^{N} *\left(\frac{1-\left(\frac{\lambda_{\alpha_{F}}^{U}\left(1+\eta_{\alpha_{F}}^{U}\right)}{\mu_{\alpha_{F}}^{U}}\right)}{1-\left(\frac{\lambda_{\alpha_{F}}^{U}\left(1+\eta_{\alpha_{F}}^{U}\right)}{\mu_{\alpha_{F}}^{U}}\right)^{N+1}}\right) \\
& =\left(\frac{\left(\lambda_{4}-\alpha_{F}\left(\lambda_{4}-\lambda_{3}\right)\right)\left(1+\left(\eta_{4}-\alpha_{F}\left(\eta_{4}-\eta_{3}\right)\right)\right)}{\left(\mu_{4}-\alpha_{F}\left(\mu_{4}-\mu_{3}\right)\right)}\right)^{N} \\
& \quad\left(\frac{1-\left(\frac{\left(\lambda_{4}-\alpha_{F}\left(\lambda_{4}-\lambda_{3}\right)\right)\left(1+\left(\eta_{4}-\alpha_{F}\left(\eta_{4}-\eta_{3}\right)\right)\right)}{\left(\mu_{4}-\alpha_{F}\left(\mu_{4}-\mu_{3}\right)\right)}\right)}{1-\left(\frac{\left(\lambda_{4}-\alpha_{F}\left(\lambda_{4}-\lambda_{3}\right)\right)\left(1+\left(\eta_{4}-\alpha_{F}\left(\eta_{4}-\eta_{3}\right)\right)\right)}{\left(\mu_{4}-\alpha_{F}\left(\mu_{4}-\mu_{3}\right)\right)}\right)^{N+1}}\right) .
\end{aligned}
$$

Vague expected system size:

$$
\begin{aligned}
& (\tilde{L})_{\alpha_{T}}^{L} \\
& =\sum_{n=1}^{N} n\left[\frac{\lambda_{\alpha_{T}}^{L}\left(1+\eta_{\alpha_{T}}^{L}\right)}{\mu_{\alpha_{T}}^{L}}\right]^{n} *\left(\frac{1-\left(\frac{\lambda_{\alpha_{T}}^{L}\left(1+\eta_{\alpha_{T}}^{L}\right)}{\mu_{\alpha_{T}}^{L}}\right)}{1-\left(\frac{\lambda_{\alpha_{T}}^{L}\left(1+\eta_{\alpha_{T}}^{L}\right)}{\mu_{\alpha_{T}}^{L}}\right)^{N+1}}\right)
\end{aligned}
$$




$$
\begin{aligned}
= & \sum_{n=1}^{N} n\left[\left(\frac{\left(w \alpha_{T}\left(\lambda_{2}-\lambda_{1}\right)+\lambda_{1}\right)\left(1+\left(w \alpha_{T}\left(\eta_{2}-\eta_{1}\right)+\eta_{1}\right)\right)}{\left(w \alpha_{T}\left(\mu_{2}-\mu_{1}\right)+\mu_{1}\right)}\right)\right]^{n} \\
& *\left(\frac{1-\left(\frac{\left(w \alpha_{T}\left(\lambda_{2}-\lambda_{1}\right)+\lambda_{1}\right)\left(1+\left(w \alpha_{T}\left(\eta_{2}-\eta_{1}\right)+\eta_{1}\right)\right)}{\left(w \alpha_{T}\left(\mu_{2}-\mu_{1}\right)+\mu_{1}\right)}\right)}{1-\left(\frac{\left(w \alpha_{T}\left(\lambda_{2}-\lambda_{1}\right)+\lambda_{1}\right)\left(1+\left(w \alpha_{T}\left(\eta_{2}-\eta_{1}\right)+\eta_{1}\right)\right)}{\left(w \alpha_{T}\left(\mu_{2}-\mu_{1}\right)+\mu_{1}\right)}\right)^{N+1}}\right),
\end{aligned}
$$

$$
\begin{aligned}
& (\tilde{L})_{\alpha_{T}}^{U} \\
& =\sum_{n=1}^{N} n\left[\frac{\lambda_{\alpha_{T}}^{U}\left(1+\eta_{\alpha_{T}}^{U}\right)}{\mu_{\alpha_{T}}^{U}}\right]^{n} *\left(\frac{1-\left(\frac{\lambda_{\alpha_{T}}^{U}\left(1+\eta_{\alpha_{T}}^{U}\right)}{\mu_{\alpha_{T}}^{U}}\right)}{1-\left(\frac{\lambda_{\alpha_{T}}^{U}\left(1+\eta_{\alpha_{T}}^{U}\right)}{\mu_{\alpha_{T}}^{U}}\right)^{N+1}}\right) \\
& =\sum_{n=1}^{N} n\left[\left(\frac{\left(\lambda_{4}-w \alpha_{T}\left(\lambda_{4}-\lambda_{3}\right)\right)\left(1+\left(\eta_{4}-w \alpha_{T}\left(\eta_{4}-\eta_{3}\right)\right)\right)}{\left(\mu_{4}-w \alpha_{T}\left(\mu_{4}-\mu_{3}\right)\right)}\right)\right]^{n} \\
& \quad *\left(\frac{1-\left(\frac{\left(\lambda_{4}-w \alpha_{T}\left(\lambda_{4}-\lambda_{3}\right)\right)\left(1+\left(\eta_{4}-w \alpha_{T}\left(\eta_{4}-\eta_{3}\right)\right)\right)}{\left(\mu_{4}-w \alpha_{T}\left(\mu_{4}-\mu_{3}\right)\right)}\right)}{1-\left(\frac{\left(\lambda_{4}-w \alpha_{T}\left(\lambda_{4}-\lambda_{3}\right)\right)\left(1+\left(\eta_{4}-w \alpha_{T}\left(\eta_{4}-\eta_{3}\right)\right)\right)}{\left(\mu_{4}-w \alpha_{T}\left(\mu_{4}-\mu_{3}\right)\right)}\right)^{N+1}}\right)
\end{aligned}
$$

$$
\begin{aligned}
& (\tilde{L})_{\alpha_{F}}^{L} \\
& =\sum_{n=1}^{N} n\left[\frac{\lambda_{\alpha_{F}}^{L}\left(1+\eta_{\alpha_{F}}^{L}\right)}{\mu_{\alpha_{F}}^{L}}\right]^{n} *\left(\frac{1-\left(\frac{\lambda_{\alpha_{F}}^{L}\left(1+\eta_{\alpha_{F}}^{L}\right)}{\mu_{\alpha_{F}}^{L}}\right)}{1-\left(\frac{\lambda_{\alpha_{F}}^{L}\left(1+\eta_{\alpha_{F}}^{L}\right)}{\mu_{\alpha_{F}}^{L}}\right)}\right) \\
& =\sum_{n=1}^{N+1} n\left[\left(\frac{\left(\alpha_{F}\left(\lambda_{2}-\lambda_{1}\right)+\lambda_{1}\right)\left(1+\left(\alpha_{F}\left(\eta_{2}-\eta_{1}\right)+\eta_{1}\right)\right)}{\left(\alpha_{F}\left(\mu_{2}-\mu_{1}\right)+\mu_{1}\right)}\right)\right]^{n} \\
& \quad *\left(\frac{1-\left(\frac{\left(\alpha_{F}\left(\lambda_{2}-\lambda_{1}\right)+\lambda_{1}\right)\left(1+\left(\alpha_{F}\left(\eta_{2}-\eta_{1}\right)+\eta_{1}\right)\right)}{\left(\alpha_{F}\left(\mu_{2}-\mu_{1}\right)+\mu_{1}\right)}\right)}{1-\left(\frac{\left(\alpha_{F}\left(\lambda_{2}-\lambda_{1}\right)+\lambda_{1}\right)\left(1+\left(\alpha_{F}\left(\eta_{2}-\eta_{1}\right)+\eta_{1}\right)\right)}{\left(\alpha_{F}\left(\mu_{2}-\mu_{1}\right)+\mu_{1}\right)}\right)}\right)
\end{aligned}
$$

$$
\begin{aligned}
& (\tilde{L})_{\alpha_{F}}^{U} \\
& =\sum_{n=1}^{N} n\left[\frac{\lambda_{\alpha_{F}}^{U}\left(1+\eta_{\alpha_{F}}^{U}\right)}{\mu_{\alpha_{F}}^{U}}\right]^{n} *\left(\frac{1-\left(\frac{\lambda_{\alpha_{F}}^{U}\left(1+\eta_{\alpha_{F}}^{U}\right)}{\mu_{\alpha_{F}}^{U}}\right)}{1-\left(\frac{\lambda_{\alpha_{F}}^{U}\left(1+\eta_{\alpha_{F}}^{U}\right)}{\mu_{\alpha_{F}}^{U}}\right)^{N+1}}\right) \\
& =\sum_{n=1}^{N} n\left[\left(\frac{\left(\lambda_{4}-\alpha_{F}\left(\lambda_{4}-\lambda_{3}\right)\right)\left(1+\left(\eta_{4}-\alpha_{F}\left(\eta_{4}-\eta_{3}\right)\right)\right)}{\left(\mu_{4}-\alpha_{F}\left(\mu_{4}-\mu_{3}\right)\right)}\right]^{n}\right. \\
& \quad *\left(\frac{1-\left(\frac{\left(\lambda_{4}-\alpha_{F}\left(\lambda_{4}-\lambda_{3}\right)\left(1+\left(\eta_{4}-\alpha_{F}\left(\eta_{4}-\eta_{3}\right)\right)\right)\right.}{\left(\mu_{4}-\alpha_{F}\left(\mu_{4}-\mu_{3}\right)\right)}\right)}{1-\left(\frac{\left(\lambda_{4}-\alpha_{F}\left(\lambda_{4}-\lambda_{3}\right)\right)\left(1+\left(\eta_{4}-\alpha_{F}\left(\eta_{4}-\eta_{3}\right)\right)\right)}{\left(\mu_{4}-\alpha_{F}\left(\mu_{4}-\mu_{3}\right)\right)}\right)^{N+1}}\right) .
\end{aligned}
$$

Vague expected number of the customers waiting in the queue is computed by:

$$
\begin{aligned}
\left(\tilde{L}_{q}\right)_{\alpha_{T}}^{L} & \sum_{n=1}^{N}(n-1)\left[\frac{\lambda_{\alpha_{T}}^{L}\left(1+\eta_{\alpha_{T}}^{L}\right)}{\mu_{\alpha_{T}}^{L}}\right]^{n} \\
& *\left(\frac{1-\left(\frac{\lambda_{\alpha_{T}}^{L}\left(1+\eta_{\alpha_{T}}^{L}\right)}{\mu_{\alpha_{T}}^{L}}\right)}{1-\left(\frac{\lambda_{\alpha_{T}}^{L}\left(1+\eta_{\alpha_{T}}^{L}\right)}{\mu_{\alpha_{T}}^{L}}\right)^{N+1}}\right) \\
= & \sum_{n=1}^{N}(n-1) \\
& *\left[\left(\frac{\left(w \alpha_{T}\left(\lambda_{2}-\lambda_{1}\right)+\lambda_{1}\right)\left(1+\left(w \alpha_{T}\left(\eta_{2}-\eta_{1}\right)+\eta_{1}\right)\right)}{\left(w \alpha_{T}\left(\mu_{2}-\mu_{1}\right)+\mu_{1}\right)}\right]^{n}\right. \\
& *\left(\frac{1-\left(\frac{\left(w \alpha_{T}\left(\lambda_{2}-\lambda_{1}\right)+\lambda_{1}\right)\left(1+\left(w \alpha_{T}\left(\eta_{2}-\eta_{1}\right)+\eta_{1}\right)\right)}{\left(w \alpha_{T}\left(\mu_{2}-\mu_{1}\right)+\mu_{1}\right)}\right)}{1-\left(\frac{\left(w \alpha_{T}\left(\lambda_{2}-\lambda_{1}\right)+\lambda_{1}\right)\left(1+\left(w \alpha_{T}\left(\eta_{2}-\eta_{1}\right)+\eta_{1}\right)\right)}{\left(w \alpha_{T}\left(\mu_{2}-\mu_{1}\right)+\mu_{1}\right)}\right)^{N+1}}\right),
\end{aligned}
$$

$$
\begin{aligned}
& \left(\tilde{L}_{q}\right)_{\alpha_{T}}^{U} \\
= & \sum_{n=1}^{N}(n-1)\left[\frac{\lambda_{\alpha_{T}}^{U}\left(1+\eta_{\alpha_{T}}^{U}\right)}{\mu_{\alpha_{T}}^{U}}\right]^{n} \\
& *\left(\frac{1-\left(\frac{\lambda_{\alpha_{T}}^{U}\left(1+\eta_{\alpha_{T}}^{U}\right)}{\mu_{\alpha_{T}}^{U}}\right)}{1-\left(\frac{\lambda_{\alpha_{T}}^{U}\left(1+\eta_{\alpha_{T}}^{U}\right)}{\mu_{\alpha_{T}}^{U}}\right)^{N+1}}\right) \\
= & \sum_{n=1}^{N}(n-1) \\
& *\left[\left(\frac{\left(\lambda_{4}-w \alpha_{T}\left(\lambda_{4}-\lambda_{3}\right)\right)\left(1+\left(\eta_{4}-w \alpha_{T}\left(\eta_{4}-\eta_{3}\right)\right)\right)}{\left(\mu_{4}-w \alpha_{T}\left(\mu_{4}-\mu_{3}\right)\right)}\right]^{n}\right. \\
& *\left(\frac{1-\left(\frac{\left(\lambda_{4}-w \alpha_{T}\left(\lambda_{4}-\lambda_{3}\right)\right)\left(1+\left(\eta_{4}-w \alpha_{T}\left(\eta_{4}-\eta_{3}\right)\right)\right)}{\left(\mu_{4}-w \alpha_{T}\left(\mu_{4}-\mu_{3}\right)\right)}\right)}{1-\left(\frac{\left(\lambda_{4}-w \alpha_{T}\left(\lambda_{4}-\lambda_{3}\right)\right)\left(1+\left(\eta_{4}-w \alpha_{T}\left(\eta_{4}-\eta_{3}\right)\right)\right)}{\left(\mu_{4}-w \alpha_{T}\left(\mu_{4}-\mu_{3}\right)\right)}\right)^{N+1}}\right),
\end{aligned}
$$

$\left(\tilde{L}_{q}\right)_{\alpha_{F}}^{L}$

$=\sum_{n=1}^{N}(n-1)\left[\frac{\lambda_{\alpha_{F}}^{L}\left(1+\eta_{\alpha_{F}}^{L}\right)}{\mu_{\alpha_{F}}^{L}}\right]^{n}$

$*\left(\frac{1-\left(\frac{\lambda_{\alpha_{F}}^{L}\left(1+\eta_{\alpha_{F}}^{L}\right)}{\mu_{\alpha_{F}}^{L}}\right)}{1-\left(\frac{\lambda_{\alpha_{F}}^{L}\left(1+\eta_{\alpha_{F}}^{L}\right)}{\mu_{\alpha_{F}}^{L}}\right)^{N+1}}\right)$

$=\sum_{n=1}^{N}(n-1)$ 


$$
\begin{aligned}
& *\left[\left(\frac{\left(\alpha_{F}\left(\lambda_{2}-\lambda_{1}\right)+\lambda_{1}\right)\left(1+\left(\alpha_{F}\left(\eta_{2}-\eta_{1}\right)+\eta_{1}\right)\right)}{\left(\alpha_{F}\left(\mu_{2}-\mu_{1}\right)+\mu_{1}\right)}\right)\right]^{n} \\
& *\left(\frac{1-\left(\frac{\left(\alpha_{F}\left(\lambda_{2}-\lambda_{1}\right)+\lambda_{1}\right)\left(1+\left(\alpha_{F}\left(\eta_{2}-\eta_{1}\right)+\eta_{1}\right)\right)}{\left(\alpha_{F}\left(\mu_{2}-\mu_{1}\right)+\mu_{1}\right)}\right)}{1-\left(\frac{\left(\alpha_{F}\left(\lambda_{2}-\lambda_{1}\right)+\lambda_{1}\right)\left(1+\left(\alpha_{F}\left(\eta_{2}-\eta_{1}\right)+\eta_{1}\right)\right)}{\left(\alpha_{F}\left(\mu_{2}-\mu_{1}\right)+\mu_{1}\right)}\right)^{N+1}}\right),
\end{aligned}
$$

$$
\begin{aligned}
\left(\tilde{L}_{q}\right)_{\alpha_{F}}^{U} & \sum_{n=1}^{N}(n-1)\left[\frac{\lambda_{\alpha_{F}}^{U}\left(1+\eta_{\alpha_{F}}^{U}\right)}{\mu_{\alpha_{F}}^{U}}\right]^{n} \\
& *\left(\frac{1-\left(\frac{\lambda_{\alpha_{F}}^{U}\left(1+\eta_{\alpha_{F}}^{U}\right)}{\mu_{\alpha_{F}}^{U}}\right)}{1-\left(\frac{\lambda_{\alpha_{F}}^{U}\left(1+\eta_{\alpha_{F}}^{U}\right)}{\mu_{\alpha_{F}}^{U}}\right)^{N+1}}\right) \\
= & \sum_{n=1}^{N}(n-1) \\
& *\left[\left(\frac{\left(\lambda_{4}-\alpha_{F}\left(\lambda_{4}-\lambda_{3}\right)\right)\left(1+\left(\eta_{4}-\alpha_{F}\left(\eta_{4}-\eta_{3}\right)\right)\right)}{\left(\mu_{4}-\alpha_{F}\left(\mu_{4}-\mu_{3}\right)\right)}\right]^{n}\right. \\
& *\left(\frac{\left(\frac{\left(\lambda_{4}-\alpha_{F}\left(\lambda_{4}-\lambda_{3}\right)\right)\left(1+\left(\eta_{4}-\alpha_{F}\left(\eta_{4}-\eta_{3}\right)\right)\right.}{\left(\mu_{4}-\alpha_{F}\left(\mu_{4}-\mu_{3}\right)\right)}\right)}{1-\left(\frac{\left(\lambda_{4}-\alpha_{F}\left(\lambda_{4}-\lambda_{3}\right)\right)\left(1+\left(\eta_{4}-\alpha_{F}\left(\eta_{4}-\eta_{3}\right)\right)\right)}{\left(\mu_{4}-\alpha_{F}\left(\mu_{4}-\mu_{3}\right)\right)}\right)^{N+1}}\right) .
\end{aligned}
$$

The membership functions for $\tilde{L}, \tilde{L}_{q}$ :

$$
\begin{gathered}
T_{\tilde{L}}(L)= \begin{cases}\frac{L-L_{1}}{w\left(L_{2}-L_{1}\right)}, & L_{1} \leq L \leq L_{2}, \\
1, & L_{2} \leq L \leq L_{3}, \\
\frac{L_{4}-L}{w\left(L_{4}-L_{3}\right)}, & L_{3} \leq L \leq L_{4}, \\
0, & \text { otherwise, }\end{cases} \\
1-F_{\tilde{L}}(L)= \begin{cases}\frac{L-L_{1}}{\left(L_{2}-L_{1}\right)}, & L_{1} \leq L \leq L_{2}, \\
\frac{L_{4}-L}{\left(L_{4}-L_{3}\right)}, & L_{3} \leq L \leq L_{4}, \\
0, & \text { otherwise, }\end{cases} \\
T_{\tilde{L_{q}}}\left(L_{q}\right)= \begin{cases}\frac{L_{q}-L_{q_{1}}}{w\left(L_{q_{2}}-L_{q_{1}}\right)}, & L_{q_{1}} \leq L_{q} \leq L_{q_{2}}, \\
1, & L_{q_{2}} \leq L_{q} \leq L_{q_{3}}, \\
\frac{L_{q_{4}}-L_{q}}{w\left(L_{q_{4}}-L_{q_{3}}\right)}, & L_{q_{3}} \leq L_{q} \leq L_{q_{4}}, \\
0, & \text { otherwise, }\end{cases}
\end{gathered}
$$

\section{Economic Analysis}

Economic analysis of the proposed model leads to strategically determine the role of decision making for organizations and its ability and capacity. It is required to optimize the queuing system. In other words, for an optimal system, it is needed to explore a system in which the total cost is minimized.

Here, we present the cost model:

Total Cost $=C_{S} * C+C_{h} * L+C_{l} \lambda \mathrm{P}_{n}+C_{W} * L_{q}$

$$
+C_{I} *\left(m-L+L_{q}\right),
$$

Total expected cost of the system

$$
\begin{aligned}
= & C_{S} * \mu+C_{h} \sum_{n=1}^{N} n\left[\frac{\lambda(1+\eta)}{\mu}\right]^{n} *\left(\frac{1-\left(\frac{\lambda(1+\eta)}{\mu}\right)}{1-\left(\frac{\lambda(1+\eta)}{\mu}\right)^{N+1}}\right) \\
& +C_{l} \lambda\left[\frac{\lambda(1+\eta)}{\mu}\right]^{N} *\left(\frac{1-\left(\frac{\lambda(1+\eta)}{\mu}\right)}{1-\left(\frac{\lambda(1+\eta)}{\mu}\right)^{N+1}}\right) \\
& +C_{w} \sum_{n=1}^{N}(n-1)\left[\frac{\lambda(1+\eta)}{\mu}\right]^{n} *\left(\frac{1-\left(\frac{\lambda(1+\eta)}{\mu}\right)}{1-\left(\frac{\lambda(1+\eta)}{\mu}\right)^{N+1}}\right) \\
& +C_{I} *\left(m-\left(\sum_{n=1}^{N} n\left[\frac{\lambda(1+\eta)}{\mu}\right]^{n} *\left(\frac{1-\left(\frac{\lambda(1+\eta)}{\mu}\right)}{1-\left(\frac{\lambda(1+\eta)}{\mu}\right)^{N+1}}\right)\right)\right.
\end{aligned}
$$




$$
\left.+\left(\sum_{n=1}^{N}(n-1)\left[\frac{\lambda(1+\eta)}{\mu}\right]^{n} *\left(\frac{1-\left(\frac{\lambda(1+\eta)}{\mu}\right)}{1-\left(\frac{\lambda(1+\eta)}{\mu}\right)^{N+1}}\right)\right)\right) .
$$

Let:

$C_{W}$ : Cost of one customer waiting in queue,

$C_{S}:$ Cost per service per unit time,

$C_{h}$ : Holding cost per unit per unit time,

$C_{l}$ : Cost of cancellation per customer per unit time in queue,

$C_{I}$ : Cost of keeping an idle server in a unit of time,

$M$ : Number of the servers.

Vague total expected cost of the system:

$$
\begin{aligned}
& (T E C)_{\alpha_{T}}^{L} \\
& =\left(C_{S} * \mu_{\alpha_{T}}^{L}\right)+C_{h} *\left(\sum_{n=1}^{N} n\left[\frac{\lambda_{\alpha_{T}}^{L}\left(1+\eta_{\alpha_{T}}^{L}\right)}{\mu_{\alpha_{T}}^{L}}\right]^{n}\right. \\
& \text { *( } \left.\left(\frac{1-\left(\frac{\lambda_{\alpha_{T}}^{L}\left(1+\eta_{\alpha_{T}}^{L}\right)}{\mu_{\alpha_{T}}^{L}}\right)}{1-\left(\frac{\lambda_{\alpha_{T}}^{L}\left(1+\eta_{\alpha_{T}}^{L}\right)}{\mu_{\alpha_{T}}^{L}}\right)^{N+1}}\right)\right) \\
& +\left(C_{l} * \lambda_{\alpha_{T}}^{L}\right) *\left(\left[\frac{\lambda_{\alpha_{T}}^{L}\left(1+\eta_{\alpha_{T}}^{L}\right)}{\mu_{\alpha_{T}}^{L}}\right]^{N}\right. \\
& \text { *( } \left.\left(\frac{1-\left(\frac{\lambda_{\alpha_{T}}^{L}\left(1+\eta_{\alpha_{T}}^{L}\right)}{\mu_{\alpha_{T}}^{L}}\right)}{1-\left(\frac{\lambda_{\alpha_{T}}^{L}\left(1+\eta_{\alpha_{T}}^{L}\right)}{\mu_{\alpha_{T}}^{L}}\right)^{N+1}}\right)\right) \\
& +C_{W} *\left(\sum_{n=1}^{N}(n-1)\left[\frac{\lambda_{\alpha_{T}}^{L}\left(1+\eta_{\alpha_{T}}^{L}\right)}{\mu_{\alpha_{T}}^{L}}\right]^{n}\right. \\
& \text { *( } \left.\left(\frac{1-\left(\frac{\lambda_{\alpha_{T}}^{L}\left(1+\eta_{\alpha_{T}}^{L}\right)}{\mu_{\alpha_{T}}^{L}}\right)}{1-\left(\frac{\lambda_{\alpha_{T}}^{L}\left(1+\eta_{\alpha_{T}}^{L}\right)}{\mu_{\alpha_{T}}^{L}}\right)^{N+1}}\right)\right) \\
& +C_{I} *\left(m-\left(\sum_{n=1}^{N} n\left[\frac{\lambda_{\alpha_{T}}^{L}\left(1+\eta_{\alpha_{T}}^{L}\right)}{\mu_{\alpha_{T}}^{L}}\right]^{n}\right.\right. \\
& \text { *( } \left.\left(\frac{1-\left(\frac{\lambda_{\alpha_{T}}^{L}\left(1+\eta_{\alpha_{T}}^{L}\right)}{\mu_{\alpha_{T}}^{L}}\right)}{1-\left(\frac{\lambda_{\alpha_{T}}^{L}\left(1+\eta_{\alpha_{T}}^{L}\right)}{\mu_{\alpha_{T}}^{L}}\right)^{N+1}}\right)\right) \\
& +\left(\sum_{n=1}^{N}(n-1)\left[\frac{\lambda_{\alpha_{T}}^{L}\left(1+\eta_{\alpha_{T}}^{L}\right)}{\mu_{\alpha_{T}}^{L}}\right]^{n}\right.
\end{aligned}
$$

*( $\left.\left(\frac{1-\left(\frac{\lambda_{\alpha_{T}}^{L}\left(1+\eta_{\alpha_{T}}^{L}\right)}{\mu_{\alpha_{T}}^{L}}\right)}{1-\left(\frac{\lambda_{\alpha_{T}}^{L}\left(1+\eta_{\alpha_{T}}^{L}\right)}{\mu_{\alpha_{T}}^{L}}\right)^{N+1}}\right)\right)$,

$(T E C)_{\alpha_{T}}^{U}$

$$
=\left(C_{S} * \mu_{\alpha_{T}}^{U}\right)+C_{h} *\left(\sum_{n=1}^{N} n\left[\frac{\lambda_{\alpha_{T}}^{U}\left(1+\eta_{\alpha_{T}}^{U}\right)}{\mu_{\alpha_{T}}^{U}}\right]^{n}\right.
$$$$
\text { *( } \left.\left(\frac{1-\left(\frac{\lambda_{\alpha_{T}}^{U}\left(1+\eta_{\alpha_{T}}^{U}\right)}{\mu_{\alpha_{T}}^{U}}\right)}{1-\left(\frac{\lambda_{\alpha_{T}}^{U}\left(1+\eta_{\alpha_{T}}^{U}\right)}{\mu_{\alpha_{T}}^{U}}\right)^{N+1}}\right)\right)
$$$$
+\left(C_{l} * \lambda_{\alpha_{T}}^{U}\right) *\left(\left[\frac{\lambda_{\alpha_{T}}^{U}\left(1+\eta_{\alpha_{T}}^{U}\right)}{\mu_{\alpha_{T}}^{U}}\right]^{N}\right.
$$$$
\left.*\left(\frac{1-\left(\frac{\lambda_{\alpha_{T}}^{U}\left(1+\eta_{\alpha_{T}}^{U}\right)}{\mu_{\alpha_{T}}^{U}}\right)}{1-\left(\frac{\lambda_{\alpha_{T}}^{U}\left(1+\eta_{\alpha_{T}}^{U}\right)}{\mu_{\alpha_{T}}^{U}}\right)^{N+1}}\right)\right)
$$$$
+C_{W} *\left(\sum_{n=1}^{N}(n-1)\left[\frac{\lambda_{\alpha_{T}}^{U}\left(1+\eta_{\alpha_{T}}^{U}\right)}{\mu_{\alpha_{T}}^{U}}\right]^{n}\right.
$$$$
\text { *( } \left.\left(\frac{1-\left(\frac{\lambda_{\alpha_{T}}^{U}\left(1+\eta_{\alpha_{T}}^{U}\right)}{\mu_{\alpha_{T}}^{U}}\right)}{1-\left(\frac{\lambda_{\alpha_{T}}^{U}\left(1+\eta_{\alpha_{T}}^{U}\right)}{\mu_{\alpha_{T}}^{U}}\right)^{N+1}}\right)\right)
$$$$
+C_{I} *\left(m-\left(\sum_{n=1}^{N} n\left[\frac{\lambda_{\alpha_{T}}^{U}\left(1+\eta_{\alpha_{T}}^{U}\right)}{\mu_{\alpha_{T}}^{U}}\right]^{n}\right.\right.
$$$$
\text { *( } \left.\left(\frac{1-\left(\frac{\lambda_{\alpha_{T}}^{U}\left(1+\eta_{\alpha_{T}}^{U}\right)}{\mu_{\alpha_{T}}^{U}}\right)}{1-\left(\frac{\lambda_{\alpha_{T}}^{U}\left(1+\eta_{\alpha_{T}}^{U}\right)}{\mu_{\alpha_{T}}^{U}}\right)^{N+1}}\right)\right)
$$$$
+\left(\sum_{n=1}^{N}(n-1)\left[\frac{\lambda_{\alpha_{T}}^{U}\left(1+\eta_{\alpha_{T}}^{U}\right)}{\mu_{\alpha_{T}}^{U}}\right]^{n}\right.
$$$$
\text { *( } \left.\left(\frac{1-\left(\frac{\lambda_{\alpha_{T}}^{U}\left(1+\eta_{\alpha_{T}}^{U}\right)}{\mu_{\alpha_{T}}^{U}}\right)}{1-\left(\frac{\lambda_{\alpha_{T}}^{U}\left(1+\eta_{\alpha_{T}}^{U}\right)}{\mu_{\alpha_{T}}^{U}}\right)^{N+1}}\right)\right),
$$

$(T E C)_{\alpha_{F}}^{L}$

$$
\begin{aligned}
= & \left(C_{S} * \mu_{\alpha_{F}}^{L}\right)+C_{h} *\left(\sum_{n=1}^{N} n\left[\frac{\lambda_{\alpha_{F}}^{L}\left(1+\eta_{\alpha_{F}}^{L}\right)}{\mu_{\alpha_{F}}^{L}}\right]^{n}\right. \\
& \left.*\left(\frac{1-\left(\frac{\lambda_{\alpha_{F}}^{L}\left(1+\eta_{\alpha_{F}}^{L}\right)}{\mu_{\alpha_{F}}^{L}}\right)}{1-\left(\frac{\lambda_{\alpha_{F}}^{L}\left(1+\eta_{\alpha_{F}}^{L}\right)}{\mu_{\alpha_{F}}^{L}}\right)^{N+1}}\right)\right)
\end{aligned}
$$




$$
\begin{aligned}
& +\left(C_{l} * \lambda_{\alpha_{F}}^{L}\right) *\left(\left[\frac{\lambda_{\alpha_{F}}^{L}\left(1+\eta_{\alpha_{F}}^{L}\right)}{\mu_{\alpha_{F}}^{L}}\right]^{N}\right. \\
& \left.*\left(\frac{1-\left(\frac{\lambda_{\alpha_{F}}^{L}\left(1+\eta_{\alpha_{F}}^{L}\right)}{\mu_{\alpha_{F}}^{L}}\right)}{1-\left(\frac{\lambda_{\alpha_{F}}^{L}\left(1+\eta_{\alpha_{F}}^{L}\right)}{\mu_{\alpha_{F}}^{L}}\right)^{N+1}}\right)\right) \\
& +C_{W} *\left(\sum_{n=1}^{N}(n-1)\left[\frac{\lambda_{\alpha_{F}}^{L}\left(1+\eta_{\alpha_{F}}^{L}\right)}{\mu_{\alpha_{F}}^{L}}\right]^{n}\right. \\
& \left.*\left(\frac{1-\left(\frac{\lambda_{\alpha_{F}}^{L}\left(1+\eta_{\alpha_{F}}^{L}\right)}{\mu_{\alpha_{F}}^{L}}\right)}{1-\left(\frac{\lambda_{\alpha_{F}}^{L}\left(1+\eta_{\alpha_{F}}^{L}\right)}{\mu_{\alpha_{F}}^{L}}\right)^{N+1}}\right)\right) \\
& +C_{I} *\left(m-\left(\sum_{n=1}^{N} n\left[\frac{\lambda_{\alpha_{F}}^{L}\left(1+\eta_{\alpha_{F}}^{L}\right)}{\mu_{\alpha_{F}}^{L}}\right]^{n}\right.\right. \\
& \left.*\left(\frac{1-\left(\frac{\lambda_{\alpha_{F}}^{L}\left(1+\eta_{\alpha_{F}}^{L}\right)}{\mu_{\alpha_{F}}^{L}}\right)}{1-\left(\frac{\lambda_{\alpha_{F}}^{L}\left(1+\eta_{\alpha_{F}}^{L}\right)}{\mu_{\alpha_{F}}^{L}}\right)^{N+1}}\right)\right) \\
& +\left(\sum_{n=1}^{N}(n-1)\left[\frac{\lambda_{\alpha_{F}}^{L}\left(1+\eta_{\alpha_{F}}^{L}\right)}{\mu_{\alpha_{F}}^{L}}\right]^{n}\right. \\
& \left.*\left(\frac{1-\left(\frac{\lambda_{\alpha_{F}}^{L}\left(1+\eta_{\alpha_{F}}^{L}\right)}{\mu_{\alpha_{F}}^{L}}\right)}{1-\left(\frac{\lambda_{\alpha_{F}}^{L}\left(1+\eta_{\alpha_{F}}^{L}\right)}{\mu_{\alpha_{F}}^{L}}\right)^{N+1}}\right)\right), \\
& (T E C)_{\alpha_{F}}^{U} \\
& =\left(C_{S} * \mu_{\alpha_{F}}^{U}\right)+C_{h} *\left(\sum_{n=1}^{N} n\left[\frac{\lambda_{\alpha_{F}}^{U}\left(1+\eta_{\alpha_{F}}^{U}\right)}{\mu_{\alpha_{F}}^{U}}\right]^{n}\right. \\
& \left.*\left(\frac{1-\left(\frac{\lambda_{\alpha_{F}}^{U}\left(1+\eta_{\alpha_{F}}^{U}\right)}{\mu_{\alpha_{F}}^{U}}\right)}{1-\left(\frac{\lambda_{\alpha_{F}}^{U}\left(1+\eta_{\alpha_{F}}^{U}\right)}{\mu_{\alpha_{F}}^{U}}\right)^{N+1}}\right)\right) \\
& +\left(C_{l} * \lambda_{\alpha_{F}}^{U}\right) *\left(\left[\frac{\lambda_{\alpha_{F}}^{U}\left(1+\eta_{\alpha_{F}}^{U}\right)}{\mu_{\alpha_{F}}^{U}}\right]^{N}\right. \\
& \left.*\left(\frac{1-\left(\frac{\lambda_{\alpha_{F}}^{U}\left(1+\eta_{\alpha_{F}}^{U}\right)}{\mu_{\alpha_{F}}^{U}}\right)}{1-\left(\frac{\lambda_{\alpha_{F}}^{U}\left(1+\eta_{\alpha_{F}}^{U}\right)}{\mu_{\alpha_{F}}^{U}}\right)^{N+1}}\right)\right) \\
& +C_{W} *\left(\sum_{n=1}^{N}(n-1)\left[\frac{\lambda_{\alpha_{F}}^{U}\left(1+\eta_{\alpha_{F}}^{U}\right)}{\mu_{\alpha_{F}}^{U}}\right]^{n}\right. \\
& \left.*\left(\frac{1-\left(\frac{\lambda_{\alpha_{F}}^{U}\left(1+\eta_{\alpha_{F}}^{U}\right)}{\mu_{\alpha_{F}}^{U}}\right)}{1-\left(\frac{\lambda_{\alpha_{F}}^{U}\left(1+\eta_{\alpha_{F}}^{U}\right)}{\mu_{\alpha_{F}}^{U}}\right)^{N+1}}\right)\right)
\end{aligned}
$$$$
\begin{aligned}
& +C_{I} *\left(m-\left(\sum_{n=1}^{N} n\left[\frac{\lambda_{\alpha_{F}}^{U}\left(1+\eta_{\alpha_{F}}^{U}\right)}{\mu_{\alpha_{F}}^{U}}\right]^{n}\right.\right. \\
& \left.*\left(\frac{1-\left(\frac{\lambda_{\alpha_{F}}^{U}\left(1+\eta_{\alpha_{F}}^{U}\right)}{\mu_{\alpha_{F}}^{U}}\right)}{1-\left(\frac{\lambda_{\alpha_{F}}^{U}\left(1+\eta_{\alpha_{F}}^{U}\right)}{\mu_{\alpha_{F}}^{U}}\right)^{N+1}}\right)\right) \\
& +\left(\sum_{n=1}^{N}(n-1)\left[\frac{\lambda_{\alpha_{F}}^{U}\left(1+\eta_{\alpha_{F}}^{U}\right)}{\mu_{\alpha_{F}}^{U}}\right]^{n}\right. \\
& \left.*\left(\frac{1-\left(\frac{\lambda_{\alpha_{F}}^{U}\left(1+\eta_{\alpha_{F}}^{U}\right)}{\mu_{\alpha_{F}}^{U}}\right)}{1-\left(\frac{\lambda_{\alpha_{F}}^{U}\left(1+\eta_{\alpha_{F}}^{U}\right)}{\mu_{\alpha_{F}}^{U}}\right)^{N+1}}\right)\right)
\end{aligned}
$$

\section{Numerical Example}

Consider the carwash station as an example (Figure 1). Assume that due to the spatial constraints on the road, carwash only has a capacity of 6 cars for waiting in the system. The driver of the car takes an average of 2 minutes to move the car. Consider now the arrival speed of 9 cars per hour and service time of 6 minutes. Poisson distribution entry rate and distribution service rates are exponential. Also, the percentage of customer change over the past years is equal to 0.75 .

Then, we can extract the following input data (Tables 1 and 2): $\tilde{\lambda}=\tilde{9}, \tilde{\mu}=\tilde{7.5}, \tilde{\eta}=0 . \tilde{7} 5, N=7, C_{S}=15, C_{w}=13$, $C_{h}=2, C_{l}=10, C_{I}=5, W=2$.

Tables 3 to 6 show that in the sensitivity analysis for different $\alpha_{T}$ and $\alpha_{F}$, there is an increase in the average rate of entry and service rates, which is expected to rise by increasing system costs and reach the maximum at a certain level.

As the service rate increases, the cost increases quickly by

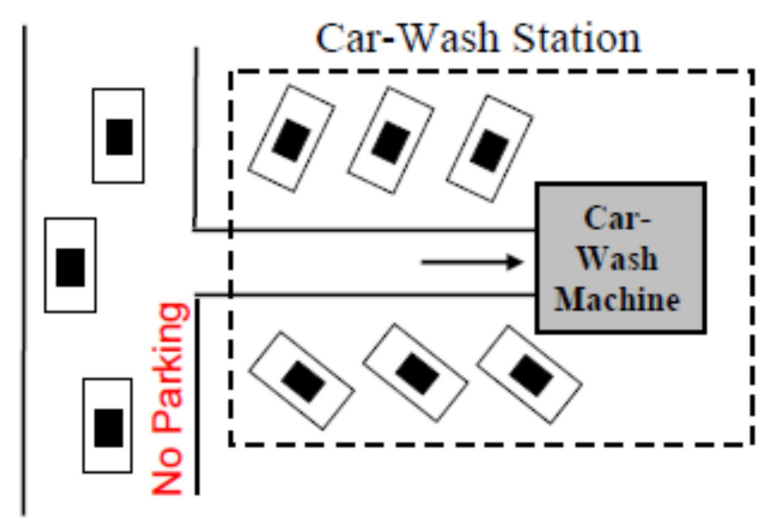

Figure 1. A carwash station. 
Table 1. Vague inputs of the problem for $\alpha_{F}$

\begin{tabular}{ccccccc}
\hline \multirow{2}{*}{$\alpha_{F}$} & \multicolumn{2}{c}{$\tilde{\lambda}$} & \multicolumn{2}{c}{$\tilde{\mu}$} & \multicolumn{2}{c}{$\tilde{\eta}$} \\
\cline { 2 - 6 } & $(\tilde{\lambda})_{\alpha_{F}}^{L}$ & $(\tilde{\lambda})_{\alpha_{F}}^{U}$ & $(\tilde{\mu})_{\alpha_{F}}^{L}$ & $(\tilde{\mu})_{\alpha_{F}}^{U}$ & $(\tilde{\eta})_{\alpha_{F}}^{L}$ & $(\tilde{\eta})_{\alpha_{F}}^{U}$ \\
\hline 0.55 & 6.75 & 16.25 & 5.25 & 14.75 & 0.525 & 1.475 \\
0.95 & 8.75 & 14.25 & 7.25 & 12.75 & 0.725 & 1.275 \\
\hline
\end{tabular}

Table 2. Vague inputs of the problem for $\alpha_{T}$

\begin{tabular}{ccccccc}
\hline \multirow{2}{*}{$\alpha_{T}$} & \multicolumn{2}{c}{$\tilde{\lambda}$} & \multicolumn{2}{c}{$\tilde{\mu}$} & \multicolumn{2}{c}{$\tilde{\eta}$} \\
\cline { 2 - 7 } & $(\tilde{\lambda})_{\alpha_{T}}^{L}$ & $(\tilde{\lambda})_{\alpha_{T}}^{U}$ & $(\tilde{\mu})_{\alpha_{T}}^{L}$ & $(\tilde{\mu})_{\alpha_{T}}^{U}$ & $(\tilde{\eta})_{\alpha_{T}}^{L}$ & $(\tilde{\eta})_{\alpha_{T}}^{U}$ \\
\hline 0.15 & 5.5 & 17.5 & 4 & 16 & 0.4 & 1.6 \\
0.45 & 8.5 & 14.5 & 7 & 13 & 0.7 & 1.3 \\
\hline
\end{tabular}

reducing the number of lines increased customer waiting time. Also, by increasing the percentage of change in the number of customers, the length of the waiting line increases. According to the sensitivity analysis, by the increase in entry rates and service rates in $\alpha_{T}$ and $\alpha_{F}$, the potential for emptying or filling up the system is reduced.

\section{Conclusions}

In this paper, we considered the vague set to study a single server queuing model with encouraged arrivals. The arrival rate and service rate were vague numbers. conducted action to exert uncertainty of the primary information when some of the parameters of the models are vague. The system can also be studied for non-heterogeneous service. The yield of the paper is of huge use for any organization exposed the phenomenon of encouraged customers augment numbers of lines and service station number inducement the time of customer queuing is cutback, and the customer satisfaction is increased load on service. By pursue and enforcement this model and useful strategy can be plotted the economic analysis can be computed and cost aspect of the business can also be beholden. To study the model validity, the mentioned model is analyzed in different scenarios of costs by the sensitivity analysis on the models' fuzzy vague costs. Regarding the conditions of the system, the obtained results can help the decision maker according to the costs of the system.

\section{References}

[1] A. K. Erlang, "The theory of probabilities and telephone conversations," Nyt Tidsskrift for Matematik Afdeling B, vol. 20, pp. 33-39, 1909.

[2] R. J. Li and E. S. Lee, "Analysis of fuzzy queues," Computers \& Mathematics with Applications, vol. 17, no. 7, pp. 1143-1147, 1989. https://doi.org/10.1016/08981221(89)90044-8

[3] J. J. Buckley, "Elementary queueing theory based on possibility theory," Fuzzy Sets and Systems, vol. 37, no. 1, pp. 43-52, 1990. https://doi.org/10.1016/0165-0114(90) 90062-B

[4] D. S. Negi and E. S. Lee, "Analysis and simulation of fuzzy queues," Fuzzy Sets and Systems, vol. 46, no. 3, pp. 321-330, 1992. https://doi.org/10.1016/0165-0114(92) 90370-J

[5] C. Kao, C. C. Li, and S. P. Chen, "Parametric programming to the analysis of fuzzy queues," Fuzzy Sets and Systems, vol. 107, no. 1, pp. 93-100, 1999. https: //doi.org/10.1016/S0165-0114(97)00295-9

[6] S. P. Chen, "Parametric nonlinear programming approach to fuzzy queues with bulk service," European Journal of Operational Research, vol. 163, no. 2, pp. 434-444, 2005. https://doi.org/10.1016/j.ejor.2003.10.041

[7] S. P. Chen, "A mathematical programming approach to the machine interference problem with fuzzy parameters," Applied Mathematics and Computation, vol. 174, no. 1, pp. 374-387, 2006. https://doi.org/10.1016/j.amc.2005.05.012

[8] L. A. Zadeh, "Fuzzy sets as a basis for a theory of possibility," Fuzzy Sets and Systems, vol. 1, no. 1, pp. 3-28, 1978. https://doi.org/10.1016/0165-0114(78)90029-5

[9] W. L. Gau and D. J. Buehrer, "Vague sets," IEEE Transactions on Systems, Man, and Cybernetics, vol. 23, no. 2, pp. 610-614, 1993. http://doi.org/10.1109/21.229476

[10] V. A. Gonzalez-Lopez, R. Gholizadeh, and A. M. Shirazi, "Optimization of queuing theory based on vague environment," International Journal of Fuzzy System Applications, vol. 5, no. 1, pp. 1-26, 2016. http://doi.org/10. 4018/IJFSA.2016010101

[11] B. Oz, I. Adan, and M. Haviv, "The Mn/Gn/1 queue with vacations and exhaustive service," European Journal of Operational Research, vol. 277, no. 3, pp. 945-952, 2019. https://doi.org/10.1016/j.ejor.2019.03.016 
Table 3. Problem answers for $\alpha_{F}$

\begin{tabular}{ccccccccccccc}
\hline \multirow{2}{*}{$\alpha_{F}$} & $\mathrm{n}$ & \multicolumn{2}{c}{$\tilde{L}$} & \multicolumn{2}{c}{$\tilde{L}_{q}$} & \multicolumn{2}{c}{$\tilde{W}$} & \multicolumn{2}{c}{$\tilde{W}_{q}$} & \multicolumn{2}{c}{ TEC } \\
\cline { 3 - 11 } & & $(\tilde{L})_{\alpha_{F}}^{L}$ & $(\tilde{L})_{\alpha_{F}}^{U}$ & $\left(\tilde{L}_{q}\right)_{\alpha_{F}}^{L}$ & $\left(\tilde{L}_{q}\right)_{\alpha_{F}}^{U}$ & $(\tilde{W})_{\alpha_{F}}^{L}$ & $(\tilde{W})_{\alpha_{F}}^{U}$ & $\left(\tilde{W}_{q}\right)_{\alpha_{F}}^{L}$ & $\left(\tilde{W}_{q}\right)_{\alpha_{F}}^{U}$ & $(\widetilde{T E C})_{\alpha_{F}}^{L}(\widetilde{T E C})_{\alpha_{F}}^{U}$ \\
\hline 1 & 0.6620 & 0.7313 & 0 & 0 & 0.0980 & 0.0450 & 0 & 0 & 126.449 & 342.1611 \\
& 2 & 1.1294 & 1.3316 & 0.5647 & 0.6658 & 0.1673 & 0.0819 & 0.0684 & 0.0446 & 128.6437 & 341.1005 \\
& 3 & 1.5744 & 1.9325 & 1.0496 & 1.2883 & 0.2332 & 0.1189 & 0.1282 & 0.0861 & 133.3436 & 346.3757 \\
& 4 & 2.0289 & 2.5401 & 1.5185 & 1.9051 & 0.3005 & 0.1563 & 0.1863 & 0.1271 & 139.2323 & 353.5698 \\
& 5 & 2.4919 & 3.1611 & 1.9935 & 2.5289 & 0.3691 & 0.1945 & 0.2450 & 0.1684 & 145.7926 & 365.0194 \\
& 6 & 2.9594 & 3.6931 & 2.4661 & 3.0776 & 0.4384 & 0.2272 & 0.3047 & 0.2062 & 152.5526 & 370.5862 \\
& 7 & 3.4224 & 3.9151 & 2.9335 & 3.3558 & 0.5070 & 0.2409 & 0.3604 & 0.2418 & 155.8642 & 365.7954 \\
& 1 & 0.6752 & 0.7695 & 0 & 0 & 0.0771 & 0.0540 & 0 & 0 & 170.8044 & 296.1995 \\
& 2 & 1.1683 & 1.4847 & 0.5841 & 0.6455 & 0.1335 & 0.1041 & 0.0667 & 0.0452 & 171.8677 & 295.3987 \\
3 & 1.6437 & 2.2971 & 1.0972 & 1.2417 & 0.1878 & 0.1612 & 0.1253 & 0.0871 & 176.5098 & 300.1733 \\
4 & 2.1304 & 3.2249 & 1.5978 & 1.8288 & 0.2434 & 0.2263 & 0.1826 & 0.1283 & 182.7217 & 306.3617 \\
5 & 2.6147 & 4.2901 & 2.0918 & 2.4199 & 0.2988 & 0.3010 & 0.2390 & 0.1698 & 189.3124 & 313.1362 \\
6 & 3.1186 & 5.4166 & 2.5988 & 2.9678 & 0.3564 & 0.3801 & 0.2970 & 0.2082 & 196.6464 & 317.9944 \\
7 & 3.5491 & 6.2642 & 3.0421 & 3.2920 & 0.4056 & 0.4395 & 0.3476 & 0.2310 & 202.2230 & 314.8889 \\
\hline
\end{tabular}

Table 4. Problem answers for $\alpha_{T}$

\begin{tabular}{cccccccccccc}
\hline \multirow{2}{*}{$\alpha_{T} \quad \mathrm{n}$} & \multicolumn{2}{c}{$\tilde{L}$} & \multicolumn{3}{c}{$\tilde{L}_{q}$} & \multicolumn{2}{c}{$\tilde{W}$} & \multicolumn{2}{c}{$\tilde{W}_{q}$} & \multicolumn{2}{c}{ TEC } \\
\cline { 2 - 12 } & $(\tilde{L})_{\alpha_{T}}^{L}$ & $(\tilde{L})_{\alpha_{T}}^{U}$ & $\left(\tilde{L}_{q}\right)_{\alpha_{T}}^{L}$ & $\left(\tilde{L}_{q}\right)_{\alpha_{T}}^{U}$ & $(\tilde{W})_{\alpha_{T}}^{L}$ & $(\tilde{W})_{\alpha_{T}}^{U}$ & $\left(\tilde{W}_{q}\right)_{\alpha_{T}}^{L}$ & $\left(\tilde{W}_{q}\right)_{\alpha_{T}}^{U}$ & $(\widetilde{T E C})_{\alpha_{T}}^{L}(\widetilde{T E C})_{\alpha_{T}}^{U}$ \\
\hline 1 & 0.6579 & 0.7394 & 0 & 0 & 0.1196 & 0.0422 & 0 & 0 & 99.2108 & 372.1768 \\
& 2 & 1.1176 & 1.3546 & 0.5588 & 0.6773 & 0.2032 & 0.0774 & 0.1016 & 0.0387 & 102.4396 & 371.6551 \\
& 3 & 1.5536 & 1.9715 & 1.0357 & 1.3143 & 0.2824 & 0.1126 & 0.1883 & 0.0751 & 107.4608 & 377.7354 \\
0.15 & 4 & 1.9938 & 2.5870 & 1.4953 & 1.9402 & 0.3625 & 0.1478 & 0.2718 & 0.1108 & 113.3460 & 385.3351 \\
& 5 & 2.4450 & 3.1574 & 1.9560 & 2.5259 & 0.4445 & 0.1804 & 0.3556 & 0.1443 & 119.7680 & 391.4890 \\
& 6 & 2.9004 & 3.8018 & 2.4170 & 3.1681 & 0.5273 & 0.2172 & 0.4394 & 0.1810 & 126.3918 & 401.5004 \\
& 7 & 3.3597 & 4.2033 & 2.8798 & 3.6028 & 0.6108 & 0.2401 & 0.5236 & 0.2058 & 133.1518 & 402.3105 \\
& 1 & 0.6734 & 0.7194 & 0 & 0 & 0.0792 & 0.0496 & 0 & 0 & 165.2188 & 302.1548 \\
& 2 & 1.1630 & 1.2961 & 0.5815 & 0.6480 & 0.1368 & 0.0893 & 0.0684 & 0.0446 & 166.4055 & 301.7357 \\
& 3 & 1.635 & 1.8737 & 1.0903 & 1.2491 & 0.1924 & 0.1292 & 0.1282 & 0.0861 & 171.0527 & 307.4152 \\
& 4 & 2.1124 & 2.4586 & 1.5843 & 1.8439 & 0.2485 & 0.1695 & 0.1863 & 0.1271 & 177.0687 & 314.9314 \\
5 & 2.6033 & 3.0533 & 2.0826 & 2.4426 & 0.3062 & 0.2105 & 0.2450 & 0.1684 & 183.9279 & 323.3439 \\
6 & 3.1080 & 3.5883 & 2.5900 & 2.9902 & 0.3656 & 0.2474 & 0.3047 & 0.2062 & 191.3260 & 329.7687 \\
7 & 3.5745 & 4.0907 & 3.0638 & 3.5063 & 0.4205 & 0.2821 & 0.3604 & 0.2418 & 197.9759 & 335.5648 \\
\hline
\end{tabular}

[12] S. Dutkova, K. Achimsky, and D. Hostakova, "Simulation of queuing system of post office," Transportation Research Procedia, vol. 40, pp. 1037-1044, 2019. https://doi.org/10.1016/j.trpro.2019.07.145

[13] S. Hanczewski, M. Stasiak, and J. Weissenberg, "Queueing model of a multi-service system with elastic and adap- tive traffic," Computer Networks, vol. 147, pp. 146-161, 2018. https://doi.org/10.1016/j.comnet.2018.09.023

[14] B. Legros, "Transient analysis of a Markovian queue with deterministic rejection," Operations Research Letters, vol. 47, no. 5, pp. 391-397, 2019. https://doi.org/10.1016/j.orl. 2019.07.005 
Table 5. Problem answers for $a_{T}$ according to the system possibility

\begin{tabular}{cccccc}
\hline \multirow{2}{*}{$\alpha_{T}$} & & \multicolumn{2}{c}{$\tilde{P}_{0}$} & \multicolumn{2}{c}{$\tilde{P}_{N}$} \\
\cline { 3 - 6 } & & $\left(\tilde{P}_{0}\right)_{\alpha_{T}}^{L}$ & $\left(\tilde{P}_{0}\right)_{\alpha_{T}}^{U}$ & $\left(\tilde{P}_{N}\right)_{\alpha_{T}}^{L}$ & $\left(\tilde{P}_{N}\right)_{\alpha_{T}}^{U}$ \\
\hline \multirow{4}{*}{0.15} & 1 & 0.3418 & 0.2601 & 0.6579 & 0.7394 \\
& 2 & 0.1508 & 0.0838 & 0.5588 & 0.6773 \\
& 3 & 0.0726 & 0.0286 & 0.5178 & 0.6571 \\
& 4 & 0.0363 & 0.0099 & 0.4984 & 0.6467 \\
& 5 & 0.0185 & 0.0034 & 0.4890 & 0.6314 \\
& 6 & 0.0095 & 0.0012 & 0.4834 & 0.6336 \\
& 7 & 0.0049 & 0.0004 & 0.4799 & 0.6004 \\
& 1 & 0.3263 & 0.2805 & 0.6734 & 0.7194 \\
& 2 & 0.1365 & 0.0985 & 0.5815 & 0.6480 \\
& 3 & 0.0620 & 0.0370 & 0.5451 & 0.6245 \\
& 4 & 0.0291 & 0.0142 & 0.5281 & 0.6146 \\
& 5 & 0.0139 & 0.0055 & 0.5206 & 0.6106 \\
& 6 & 0.0067 & 0.0021 & 0.5180 & 0.5980 \\
& 7 & 0.0032 & 0.0008 & 0.5106 & 0.5843 \\
\hline
\end{tabular}

Table 6. Problem answers for $a_{F}$ according to the system possibility

\begin{tabular}{cccccc}
\hline \multirow{2}{*}{$\alpha_{F}$} & $\mathrm{n}$ & \multicolumn{2}{c}{$\tilde{P}_{0}$} & \multicolumn{2}{c}{$\tilde{P}_{N}$} \\
\cline { 3 - 6 } & & $\left(\tilde{P}_{0}\right)_{\alpha_{F}}^{L}$ & $\left(\tilde{P}_{0}\right)_{\alpha_{F}}^{U}$ & $\left(\tilde{P}_{N}\right)_{\alpha_{F}}^{L}$ & $\left(\tilde{P}_{N}\right)_{\alpha_{F}}^{U}$ \\
\hline \multirow{4}{*}{0.55} & 1 & 0.3378 & 0.2683 & 0.6620 & 0.7313 \\
& 2 & 0.1470 & 0.0896 & 0.5647 & 0.6658 \\
& 3 & 0.0697 & 0.0318 & 0.5248 & 0.6441 \\
& 4 & 0.0343 & 0.0115 & 0.5072 & 0.6350 \\
& 5 & 0.0172 & 0.0042 & 0.4983 & 0.6322 \\
& 6 & 0.0087 & 0.0015 & 0.4932 & 0.6155 \\
& 7 & 0.0044 & 0.0005 & 0.4889 & 0.5593 \\
& 1 & 0.3245 & 0.2823 & 0.6752 & 0.7176 \\
& 2 & 0.1349 & 0.0999 & 0.5841 & 0.6455 \\
& 3 & 0.0608 & 0.0378 & 0.5479 & 0.6208 \\
& 4 & 0.0284 & 0.0146 & 0.5326 & 0.6096 \\
& 5 & 0.0134 & 0.0057 & 0.5229 & 0.6049 \\
& 6 & 0.0064 & 0.0022 & 0.5197 & 0.5935 \\
& 7 & 0.0030 & 0.0008 & 0.5070 & 0.5486 \\
\hline
\end{tabular}

[15] N. K. Jain, R. Kumar, B. K. Som, and M. An, "An M/M/1/N Queuing system with reverse balking," American Journal of Operational Research, vol. 4, no. 2, pp. 17-20, 2014. http://doi.org/10.5923/j.ajor.20140402.01

[16] R. Kumar, "A single-server Markovian queuing system with discouraged arrivals and retention of reneged cus- tomers," Yugoslav Journal of Operations Research, vol. 24, no. 1, pp. 119-126, 2016. http://doi.org/10.2298/ YJOR120911019K

[17] B. K. Som and S. Seth, "An M/M/1/N queuing system with encouraged arrivals," Global Journal of Pure and Applied Mathematics, vol. 13, no. 7, pp. 3443-3453, 2017.

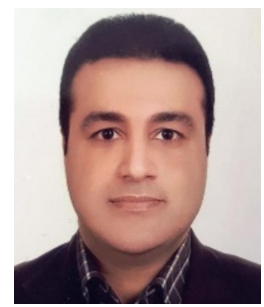

Hamed Fazlollahtabar earned a BSc and an MSc in Industrial Engineering from Mazandaran University of Science and Technology, Iran, in 2008 and 2010, respectively. He received his $\mathrm{PhD}$ in Industrial and Systems Engineering from Iran University of Science and Technology in 2015, and completed a postdoctoral research fellowship at Sharif University of Technology, Iran, in the area of reliability engineering for complex systems in 2017. He currently works in the Department of Industrial Engineering at Damghan University, Iran, and is on the editorial boards of several journals and on the technical committees of several conferences. His research interests are advanced robotic production systems, reliability engineering, supply chain planning, and business intelligence and analytics. He has published more than 280 research papers and eight books.

E-mail: hfazl@du.ac.ir

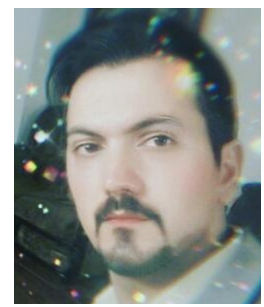

Hadi Gholizadeh was born in Amol in Mazandaran province of Iran. He earned his BSc from Shomal University, Amol, Iran and MSc from Mazandaran University of Science and Technology, Babol, Iran (MUST).His researches are mainly about Metaheuristics and Optimization techniques to solve the single and multi-objective optimization problems and also industrial engineering issues including Closed-loop Supply Chain Network Design, Sustainable developments for these supply chain systems and health care networks and also related multilevel programming models in all mentioned contents and Maintenance Engineering, Queuing systems and Machin learning and Fuzzy Computing and MCDM. He has also published more than 15 papers in leading scientific and engineering conferences e.g., IEEE and journals e.g., ASOC, IJAMT, NCCA, IJFS. 